\begin{tabular}{l} 
SCIENCE \& TECHNOLOGY \\
Journal homepage: http://www.pertanika.upm.edu.my/ \\
\hline PERTANIKA
\end{tabular}

\title{
Logistics and Freight Transportation Management: An NLP based Approach for Shipment Tracking
}

\author{
Rachit Garg ${ }^{1,2 *}$, Arvind Wamanrao Kiwelekar ${ }^{1}$ and Laxman Damodar Netak ${ }^{1}$ \\ ${ }^{1}$ Department of Computer Engineering, Dr. Babasaheb Ambedkar Technological University, \\ Lonere Maharashtra, India \\ ${ }^{2}$ Center for Advanced Technologies and Innovation, ATA Freight Line India Pvt. Ltd., Pune, Maharashtra, India
}

\begin{abstract}
Tracking and tracing systems have become basic services for most logistics companies and are particularly essential for the shipping and logistics industry. Dynamic logistics management today need constant supervision and management of continuously-changing supply chains that motivate the necessity of goods-centric logistics monitoring and tracking, which guarantees a chance to improve transparency and control of a company's multiple logistical activities. However, operational inefficiencies due to the conventional monitoring system for the supply chain management can also result in sales loss, higher cost, poor customer service-and eventually lower profits. Based on research literature, this paper aims to provide a novel approach for tracking and tracing shipment in a logistics organisation by implementing deep natural language processing concepts. The study aims to allow the stakeholders to think in new ways in their organisation and helping them to have a powerful influence on tracking and tracing to make the best decision possible at the right time. The proposed method is compared based on the accuracy of identifying the query, and results are significantly acceptable. This study is of related interest to researchers, academicians, and practitioners.
\end{abstract}

ARTICLE INFO

Article history:

Received: 19 April 2021

Accepted: 15 July 2021

Published: 18 October 2021

DOI: https://doi.org/10.47836/pjst.29.4.28

E-mail addresses:

rachit.garg.nitttr@gmail.com (Rachit Garg) awk@dbatu.ac.in (Arvind Wamanrao Kiwelekar)

1dnetak@dbatu.ac.in (Laxman Damodar Netak)

* Corresponding author
Keywords: Logistics and deep NLP, NLP, natural language processing, natural language query, speechto-text, tracking system

\section{INTRODUCTION}

The way business operates worldwide has experienced dramatic changes over the past decade, with conventional industrial, political, and geographical borders almost vanishing. The logistics sector was not left 
untouched by such changes under global market conditions. Global business logistics played a crucial role in adapting to the changing consumer demand in globalisation and mass adaptation. In times of globalisation, businesses increasingly need versatility and agility to satisfy the rising needs of adaptation and fluctuating consumer demand (Klein $\&$ Thomas, 2009). The dynamics of worldwide supply chains have ensured that their manageability has increased (Artto et al., 1998). Supply chains are becoming more complex worldwide (Christopher \& Peck, 2004). As a result, a company's success depends more and more on its supply network, and individual companies are no longer competing as independent entities but as supply chains (Lambert \& Cooper, 2000). The world in which logistics and transport managers find themselves is very different today from just a few years ago. Today's managers in the Logistics service industry have several challenges and opportunities in dramatic contrast to a decade ago. It is, therefore, no wonder that many managers have not entirely adapted to the changing world and have missed chances and success deficits. The prospect of exploiting the transport role as a crucial strategic factor in the supply chain is prominent among the list of missed opportunities. With globalisation, the nature of services, and higher consumer expectations, businesses turn their supply chain into the epicentre of industry innovation using state-of-the-art technologies.

Rising technological advances are widening industry-wide, and logistics and the supply chain can be the most affected industries. Renowned for the substantial use of manual procedures and the vast volume of data processed at various locations and in different ways, logistics has the most significant advantage from adopting emerging technology and pursuing the most creative logistics trends. As digitalisation takes hold and consumer expectancies grow, logistic companies are witnessing a period of unparalleled transition. Innovations make it possible to be more competitive and collaborative, and they re-form the market in ways that only start to emerge. Many advances in current logistics at the global and local level motivate the need for more transparency in supply chain structures or collaboration with creative logistical service providers (Klumpp et al., 2011).

The transparency of the supply chain is the ability to monitor a wide range of goods during transport to obtain a simple overview of each stage of the process. It helps businesses enhance their customer support and cost control through continuous inventory management, constructive status updating, interruption limits, and risk reduction (Gnimpieba et al., 2015). Without adequate systems, businesses face enormous challenges because the requisite supply chain transparency cannot be guaranteed (Baresi et al., 2016). Shipping and tracking are additionally centre segments of a board network like a modern supply chain management system intended to fulfil clients. Tracking shipments in service to customers is considered significant for suppliers and essential to efficient supply networks. Transportation tracking is an integral part of customer service and is essential for logistical services. The tracking system makes it easier to identify the shipment location and informs the client in due course. 
The application of information management over the entire strategy of the logistics can be improved by monitoring the carriers in real-time and tracking them.

Responses to questions like "Where is my shipment? Furthermore, "When is my shipment coming?" in logistics is usually not adequately accurate and thus reduces the productivity of logistics service providers. As logistics is dynamic and requires numerous transport modes and carriers, improving productivity and saving the supply chain's expense is essential to connect between various parties and use real-time data. Product timely delivery is a significant factor in the happiness of customers. Higher levels of customer loyalty contribute to better engagement of consumers and company repeat. Technical solutions will play a key role by increasing the distribution pace and keeping consumers aware of the product delivery plan. It can also provide the customer with a platform for tracking orders, enhancing a sense of self-sufficiency and control for a customer, and translating customer services for saving logistical organisation time and money. Using technology to gain more insight into all aspects of the industry and make data-driven decisions based on real-time information and innovation brings productivity across the logistics process. The information-sharing framework enables manufacturers to improve cooperation with their partners in real-time.

More businesses integrate technologies into their supply chain management processes to remain globally competitive and at the forefront of a complex business environment. AI is a central component of the revolution of Industry 4.0. Organisations can use artificial intelligence (AI) to help turn the unpredictable into the envisaged.

This paper aims to develop further the above concepts and apply an intelligent transport management conceptual model. The purpose of this paper is twofold: first, the analysis and presentation of the deficiencies of conventional tracking systems in logistics networks and second, to propose a new approach for constructing solutions for tracking in logistics networks. This paper is present in six sections. Section 2 discusses the theory of tracking systems by identifying the existing tracking systems from the literature. Section 3 discusses the motivation behind the use of Natural Language Processing (NLP). Section 4 discusses the method of implementation. Section 4 and 5 discuss the objective and significance of this study. Section 6 discusses the method of implementation. The study results are present in section 7 of this paper, and we finally conclude in Section 8 by drawing the future research directions.

\section{LITERATURE REVIEW}

There is rising interest in monitoring delivery and supplying consumers with tracking information. It is an essential customer service factor often viewed as industry practices instead of a possible competitive benefit for logistics service providers (LSPs). Industries spend substantial resources to provide their consumers with monitoring services (Töyrylä, 
1998). It allows consumers to trace and track transportation shipments in transit and, therefore, schedule and control their activities. Tracking means the IT-supported systems to determine object status in the manufacturing or logistic company's physical supply chain. A track-and-trace framework for managing interconnected logistical networks is essential and enhances customer support (Shamsuzzoha et al., 2013). In the early days of tracking, a communications system for calling service providers and tracking shipments is an example of technology to add value (Kerr, 1989). EDI and LMS were widespread for regulating the shipment and decision-making (Sullivan \& Fordyce, 1989). Loebbecke and Powell (1998) introduced the TRANSPO-TRACK approach for seamless tracking that also uses EDI. It is a technological solution to tracking issues in a logistics chain (Loebbecke \& Powell, 1998). Alexander's intelligent tracking system includes gathering information using RFID, GIS, GPS, and improving tracking efficiencies(Brewer et al., 1999). Chadil et al. (2008) provide the use of a model client-server in the real-time monitoring system. It is an integrated approach to detect shipment location information regularly sent to a server utilising a GPS/GPRS module. This study implements a tracking system using the GPS, GPRS, and Google Earth software for displaying locations (Chadil et al., 2008). A hybrid cargo level tracking system is an infrastructure-based solution that achieves greater precision and lower overall expenses than the existing system. The concept covers some advances and the newest generation of existing infrastructure (Yang et al., 2010).

The literature describes real-time tracking and tracing in various ways. Baresi et al. (2016) define traceability by an attribute that enables the shipment's current position to be determined. According to Shamsuzzoha et al. (2013), tracking refers to the product's positioning because it has value and risk of loss. Supply chain networks are an integral approach to coordinating material and product handling and monitoring from source to the final customer (Baresi et al., 2016).

According to Klein et al., tracking of shipments typically takes place via terrestrial systems (Klein \& Thomas, 2009). All terrestrial systems are based on and implemented on Earth, whereas satellite-based systems need spatial hardware. Terrestrial systems issue status messages only when they complete each process phase. Mainly satellite-based systems, such as GPS, monitor larger cargo units, covering longer distances (Kothris, 2001). Auto-ID technologies allow the positioning of shipments in the supply chain. According to the study, Auto-ID networks are the subsystem of the logistics system (Shamsuzzoha et al., 2013). A logistics system is an effective freight transfer accompanied by an information flow. GPS trackings are commonly accepted and used for outdoor monitoring, which many articles have already adopted (Sultana et al., 2016).

At the same time, a supply chain network manages large volumes of items. It requires a distribution strategy to minimise the related logistics and labour costs in handling customer claims (Ko et al., 2011). Barcodes, QR codes, WSN, RFID, and GPS successfully monitor 
and trace logistics. All these systems collect critical monitoring data like temperature, moisture, and position automatically and continuously (He et al., 2009; Jedermann et al., 2006). RFID technology provides certain distinct advantages to other forms of detecting systems, and for virtually any business enterprise, the potential of RFID technology can bring exciting possibilities. For product safety, visibility, and traceability, many industries use RFID technology. A mature RFID-solution technology allows structural enhancements across many logistics and supply chain systems. However, RFID systems are disadvantaged even with certain benefits as they entail high investment costs and lack data protection (Yuksel \& Yuksel, 2011). Musa et al. (2014) provide a thorough overview of product visibility in the supply chain, outlining different criteria, processes, and architectures. Figure 1 depicts the evolution of the logistics tracking system.

Researchers and standardizing organisations still look ahead aggressively to establish global monitoring strategies (Huvio et al., 2002). Company managers have described this monitoring framework to boost their business by enhancing consumer loyalty and incorporating IT. However, most tracking systems are for the advantage of a particular entity, and therefore the tracing and tracking of multi-organisational ecosystems is not available (Kärkkäinen et al., 2004). As a result, companies are only able to monitor their shipments. Larger organisations have developed their monitoring scheme that typically uses the same solution with the same problems. Many companies concentrating on designing global supply chain management solutions construct their systems around a server that acts as a central storage point for monitoring data. Blockchain is an emerging technology to address a range of logistics problems (Christidis \& Devetsikiotis, 2016; Kim \& Laskowski, 2018). It is due to blockchain's highly secure, trustworthy, unchangeable, collective repository, and global accessibility features. These characteristics are critical for logistics policymakers and provide end-to-end monitoring capability during transport,

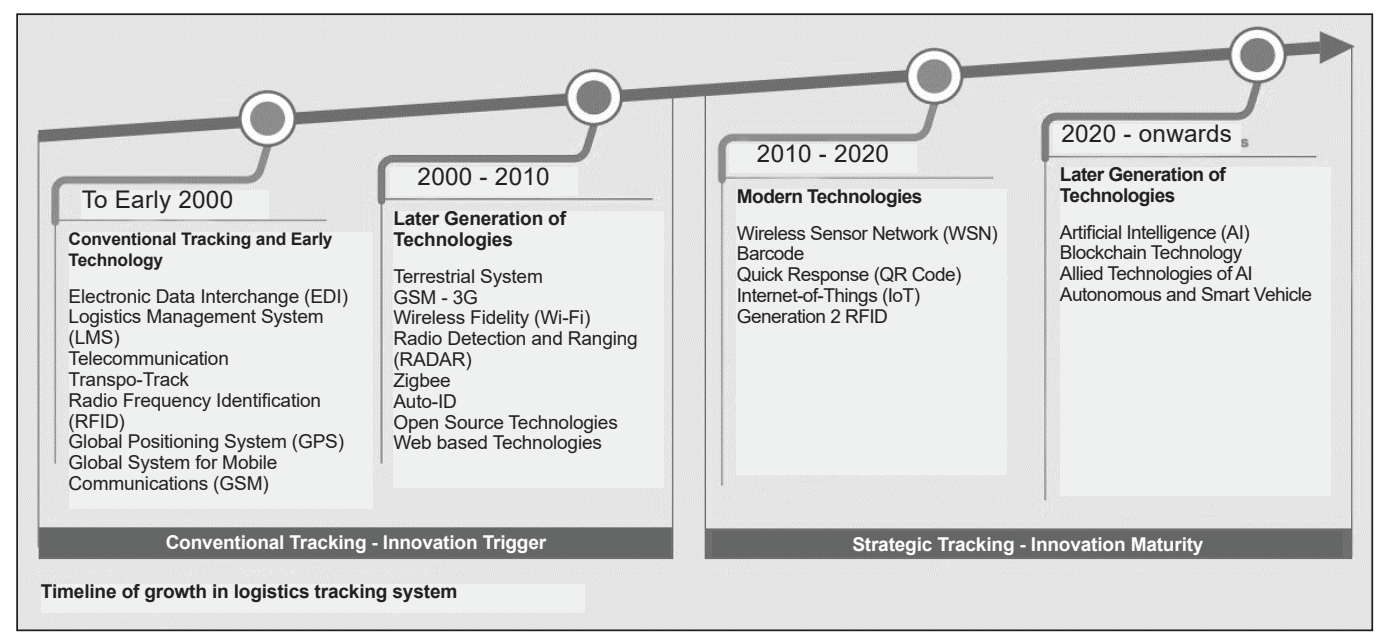

Figure 1.Timeline of growth in logistics tracking system (GEP, 2018) 
accountability, and decentralised activity without intermediary intervention (Abbas et al., 2020; Betti et al., 2019; Zhao et al., 2016).

Contemporary tracking systems may perform well if a single organisation manages products, but global supply chain networks do not fit conventional tracking approaches. Multi-company supply networks are typically carried out via a Web page to make tracking information accessible, leading to manual customer interrogation (Ruiz-Garcia et al., 2010). Automated monitoring systems should be there in the customer's operating systems for the tracking systems. It is generally, though, prolonged and sometimes time-consuming. The industry deals with various issues, including transparency in supply chains, with many end customers unaware of the origins of their shipments, complicated processes involving intermediate freight brokers, and a lack of accountability in losses is an often challenging and opaque process. Logistics and shipping networks use information systems to a different degree. A planning and tracking system must be in place to succeed in various operations and assist decision-making at different points in the supply chain.

Artificial Intelligence (AI) and its subsets are no longer science fiction; they are a digital future for many businesses. Organisations, particularly those in the logistics sector, are on the verge of implementing AI-based solutions to streamline and redefine all of their core objectives. Artificial intelligence is a broad technology phrase that refers to transforming a massive amount of data into actionable insight through algorithmic processing. For the supply chain industry, Natural Language Processing (NLP) provides various advantages. Intelligent products are eventually the secret to saving and monitoring data to the final destination. NLP is computer software that interprets, perceives, and deduces context from human language intelligently and sensitively. NLP is used to analyse text, allowing machines to understand how humans communicate. There are numerous uses of NLP in the business world, including speech comprehension, information extraction, text generation, question answering, sentiment analysis, classification, speech-to-text translation and vice versa, and knowledge acquisition (Chowdhary, 2020; Clark et al., 2010; Garg et al., 2021b; Liu et al., 2017).

NLP is an ideal tool for tackling massive amounts of valuable data. Thus, any organisation that recognises the importance of data analysis-from a single text to a collection of documents that must be summarised-will benefit from NLP. NLP capabilities transform unstructured text into actionable information. Additionally, NLP technology can assist a platform in precisely managing user expectations to boost user satisfaction effectively. NLP is now increasingly being applied to medical education, which has helped to accelerate this process over the past several years. The potential of the NLP to facilitate FOAM (Free Open-Access Medical Education) materials integration with more traditional curricular components (Chary et al., 2019).

Thessen et al. defined the use of NLP to extract biological information such as cellular processes, taxonomy names, and morphological features in their work. According to them, 
using natural language processing in biodiversity study promise to develop a semantic web for biology (Thessen et al., 2012). Biomedical and clinical text processing is a significant application area for natural language processing. Neuman et al. (2019) presented ScispaCy as a rapid and robust biomedical model. It is a specialised natural language processing library designed to address the core text processing requirements in the biomedical area (Neumann et al., 2019). In another study, Garg et al. (2021a) proposed a model for personalising news for the logistics business. It is a model that determines the importance of marine news by extracting data from corpora using statistics and deep learning methods. The study employs a natural language processing methodology to recommend news to various stakeholders in a busy media environment (Garg et al., 2021a).

\section{MOTIVATION BEHIND NLP IN LOGISTICS TRACKING}

The fourth party logistics company (4PLs) is ideal for handling all the customers' supply chains. It thus serves as their only interface to a diverse network of logistics services providers across various forms: water (sea and barge), air, road, and rail. This position poses considerable challenges for efficient freight transport planning and control while maintaining adequate service standards. Real-time monitoring data is essential to achieve this type of operational excellence.

Industries are on the verge of investing a significant budget in providing their consumers with tracking services. This monitoring and tracking enable the service providers' customers to identify the shipments in transit; thus, they schedule and control their activities. New tracking solutions may work well in managing products by one organisation, but the tracking of global supply chain networks does not follow conventional tracking approaches. Logistics organisations are spending a considerable amount on value-added services like tracing and tracking to their customers. These services play a significant role in satisfying customer demands as customers can locate shipments in transit; hence, they plan and monitor their operations. Many companies use traditional tracking approaches to handle goods by one company, but this approach might not work well to track global supply chain networks (Goll \& Bolte, 2020).

The world economy is on a stunning turnaround. Brexit and the USCMA's approval would possibly allow freight and global distribution programs to shift towards regional and more localised solutions (Bank, 2019). In 2021, global shipping technology will be a hot topic, and logistics partners need to recognise the best approach diligently. Delays and inefficiencies can vary from few days to weeks in current shipment management processes. It provides a significant opportunity and a necessity to incorporate technology and automation solutions that make timely decisions in the supply chain. This need gap requires developments from the processing of natural languages and machine learning. The Machine Learning (ML) and NLP algorithms can quickly transform large numbers of unstructured 
text into workable insights (Garg et al., 2021c). Natural language technologies have gained substantial focus from business, academia, and government since their inception. Natural language processing for the supply chain and distribution can be implemented in several respects. As supply chains produce broad data sets, it is vital to consult this data to optimise the supply chain correctly. NLP encourages users to ask difficult questions and guides them through the data to provide insights into answering them. It is also easier to simplify customer service with natural language processing. Stakeholders ask questions, and NLP responds to or directs them through the correct details. It decreases administrative costs of customer service centres and increases the supply chain's satisfaction (Garg et al., 2021b).

The automation of information-based tasks is one of the most popular and efficient NLP usage cases. The advantages of NLP range not just from operational efficiencies and productivity but also from more operational data analysis to a modest benefit. We propose an NLP-based supply chain management solution for NLP-enabled smart tracking based on the fundamental need for a pragmatic solution to the supply chain and widespread NLP technological adoption in various fields (Kota, 2019). NLP-enabled smart tracking may benefit in the following way.

\section{Monitor Shipment Movement}

NLP algorithms can monitor changes in the shipment in real-time and help to manage master data accurately.

\section{Reduce Language Barriers}

Most companies operate globally, and language barriers can impede the efficiency of processes. To minimise barriers to regional languages, NLP can translate the question from one language to another language.

\section{Tailored Stringent Delivery Expectations}

Clients want to know where their orders are and receive the most accurate delivery expectations within tighter delivery periods. In a convenient time frame, users cannot expect the quickest delivery but instead precision. These challenges go well beyond the world of consumers, as they probably have learned from corporations like Walmart, which have similar suppliers' standards.

\section{More Effective Order Tracking and Delivery}

The technology provides consistency across the entire production chain to improve insight in any aspect of the market and make real-time data-driven decisions. In supply chain management, the net effect of using technology includes decreased inventory costs, reduced 
operating capital requirements, and improved client satisfaction. For all the candidates, this is undoubtedly a victory.

In value-added supply chain management and logistics systems, industry markets today are diverse. In recent years, technological advancement has helped meet the increasing demands of tracking and tracing the logistics or transport chains. Therefore, in terms of effective logistics network management and customer demand, the value of tracking and tracing shipments is considered reasonably large for manufacturing companies (Lund et al., 2019).

\section{RESEARCH OBJECTIVE}

The study aims to examine the implementation of natural language processing in a logistics organisation to develop a natural language-based solution to tracking or tracing shipments in the logistics industry. The study's objective is to allow users to ask shipment-related questions and guide them through the data; providing workable insights to answer those questions enhances the customer experience in a logistic organisation. This research consists of a location monitoring module used to coordinate shipment tracking. Another objective of this study is to create an intelligent system that covers the core application of Natural Language Processing (NLP) to meet smart logistics services.

\section{RESEARCH SIGNIFICANCE}

Technology has a significant role to play in this new age for every industry to thrive. As the market is competitive, knowing the client base is critical. Since the prediction of consumer demand and its fulfilment has become complicated, it can be a tedious activity without good cooperation between different business partners. Supply chain management needs to provide the information of products at every stage of the logistics chain and have the correct details related to tracking the object. Each business needs to be aware of the product's physical position in the supply chain while preserving a sensitive relationship between the third party and the client and balancing costs and time. This study will help to achieve the management of transportation in the supply chain. From an academic viewpoint, the study contributes to NLP's potential in identifying information related to shipment in the logistics industry.

\section{METHOD OF IMPLEMENTATION}

The paper's work is conducted in Python. The experiment environment consists of Intel Core i5-4590 CPU, memory is $4 \mathrm{~GB}$, and the operating system is Windows 7 professional. This study is a prototype system executed on a sample dataset created from original AIS (Automatic Identification System) data. The process is described broadly in two phases: Phase one involves speech recognition and generating SQL queries from the natural 
language; Phase 2 includes the results. The second phase is mainly a DBMS system that runs the SQL query over the database. Figure 2 depicts the pictorial representation of steps in implementation. The steps of implementation are as follows:

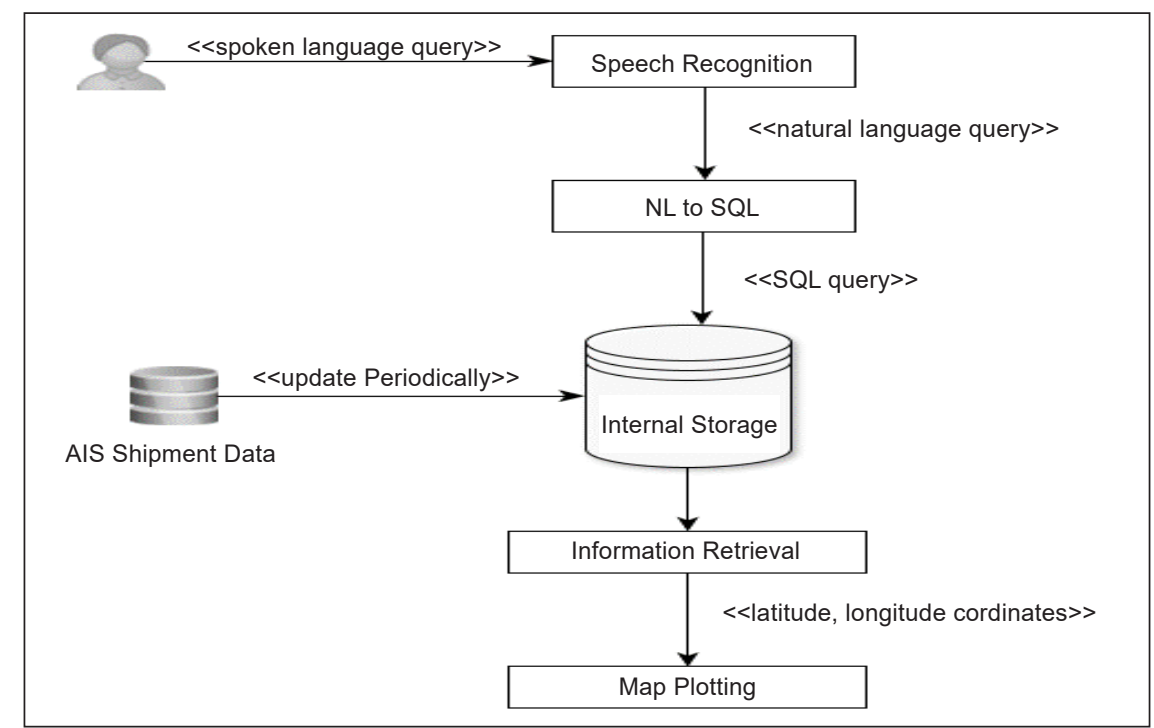

Figure 2. Steps in implementation

\section{Data Sources}

This experiment uses the dataset of shipment. The dataset is created by considering the original dataset available from AIS and all logistics companies for tracking and other shipment details. Automatic Identification Systems (AIS) monitor ship traffic to improve the safety of navigation worldwide. With these systems, planners can handle long-term movement patterns in the ocean (MarineCadastre.gov, 2013). The original dataset includes a large number of columns. Some of the primary columns are (Flag, IMO, ENI, MMSI, Call sign, Carrier, Vessel Name, Vessel Type, Time of latest Position, Destination Port, Loading Port, Built, Latitude, Longitude, Current port, global Area, Local Area, Reported Destination, Speed, Draught, Course, Navigational status, Length, Width, Capacity, Current Port Country). For this study, the original dataset is trimmed to few columns, including Port of Loading (POL), Port of Destination (POD), Carrier, Latitude, Longitude (Table 1). Another database having the serial number and status of the shipment is defined in Table 2. The serial of the 'shipment' table has a key reference with the serial of the 'status' table. The data description is given below. Once the data is collected, a machine learning or deeper learning algorithm is not recommended to be directly fitted from raw text data. First, we have to pre-process the text by passing the raw data to a series of filters implemented in Python. Once the data is preprocessed, it is available for further processing. The preprocessing of data mainly includes removing punctuation and lowering the case of data. 
Table 1

Structure of table 'shipment' for database entry

\begin{tabular}{|c|c|c|}
\hline Field Name & Data Type & Description \\
\hline Serial & Numeric & Serial no. of Shipment (key reference with status.serial) \\
\hline Shipmentid & Alphanumeric & $\begin{array}{l}\text { Shipment No. For ex. OEIN101 (Ocean Export India-Shipment } \\
\text { Serial No.) }\end{array}$ \\
\hline Mode & Alphabetic & Mode of Transportation (Ocean/Road/Air) \\
\hline Trade & Alphabetic & Trading Mode (Import/Export) \\
\hline Country & Alphabetic & Country of Trade \\
\hline Source & Alphabetic & Port of Loading (POL) \\
\hline Destination & Alphabetic & Port of Destination (POD) \\
\hline Carrier & Alphabetic & Name of the Carrier \\
\hline IsTransshipped & Boolean (Yes/No) & Movement of container from one vessel to another \\
\hline latitude & Decimal & Latitude Coordinate \\
\hline longitude & Decimal & Longitude Coordinate \\
\hline
\end{tabular}

Table 2

Structure of table 'status' for database entry

\begin{tabular}{lll}
\hline Field Name & Data Type & Description \\
\hline Serial & Numeric & Serial no. of Shipment (key reference with shipment.serial) \\
Status & Alphabetic & Status of shipment (intransit, reached, delivered) \\
\hline
\end{tabular}

\section{Speech to Natural Language Text Conversion}

The cleaned data is now ready for use with different pre-processing techniques. The primary and essential feature of any speech recognition device is text information extraction. Speech is the most popular communication, and most people rely on speech to communicate. The system of speech recognition primarily converts spoken languages into text. The deep neural network models are standard for speech-to-text conversion. Speech recognition attempts to translate a source speaker's speech into the target language text while retaining the linguistic content (Huang et al., 2019).

Speech recognition recognises spoken terms and sentences and translates them into text readable by a human being. This study uses the SpeechRecognition library to convert speech into text in a Python environment. The advantage of using this library is that no machine learning algorithm has to be developed from starting. Furthermore, this library has easy wrappers for several popular speech recognition APIs (such as Google Cloud Speech API, IBM Speech to Text). Literature shows the quick increases in implementations of speech-to-text (S2T) tasks in end-to-end sequence-to-sequence (S2S) modelling (C. Wang et al., 2020). Figure 3 shows the snapshot of implementing speech recognition to convert speech into text. 


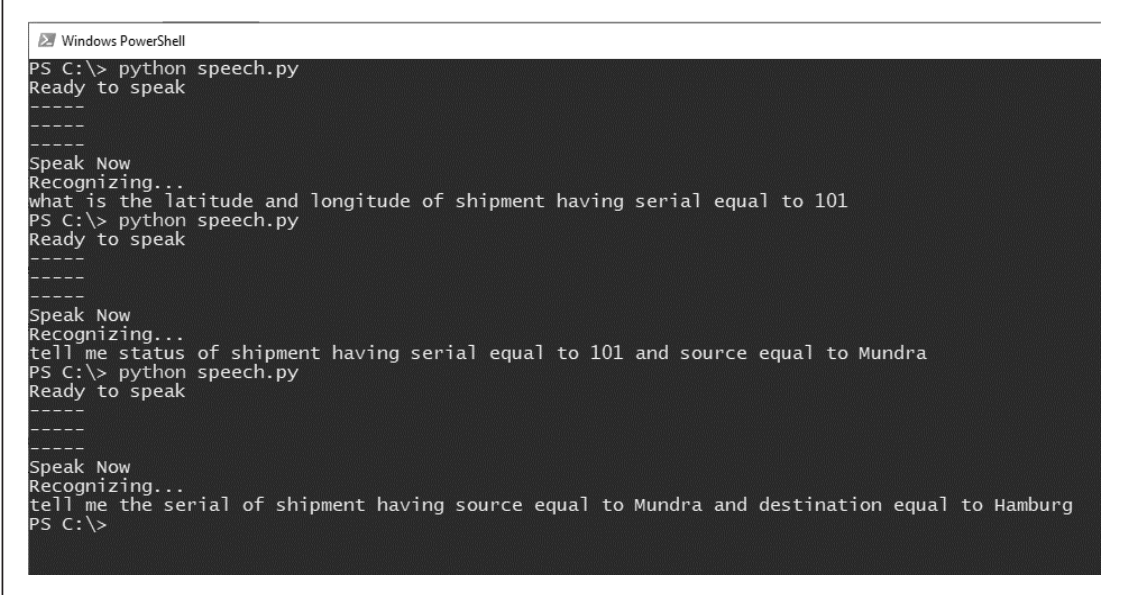

Figure 3. Speech recognition implementation to convert speech into text

\section{Converting Natural Language Text to a Query Language}

Relational databases store an immense amount of information and form the backbone of financial and customer affairs monitoring applications such as Customer Relationship Management (CRM). The Natural Language Interface (NLI), a research field on the intersection of natural language processing and human-computer interactions, aims to develop ways for people to communicate through natural language with computers (Bai et al., 2021; Li et al., 2019). This study reports one specific feature of the NLI used for relation databases: translating natural language queries into SQL. Thus, non-expert users may interact with the framework and database using NLI to the database. However, access to connection databases requires knowledge of database query language as it is essential, but for a naive user, it is challenging to expertise it (Ahkouk et al., 2020).

NLI encourages users to ask questions in a natural language without knowing the database structure or the complicated machine language. Questions entered in a natural language are translated in a formal query language in a statement (W. Wang et al., 2020). This study uses the $\ln 2 s q l$ approach for generating structured query language. $\ln 2 s q l$ is an NLP tool to convert a natural language query into a database query. This tool takes a natural language sentence as an input and converts that into a valid SQL. The other inputs for a tool include a SQL dump file for database modelling, a language configuration file for language learning, and a thesaurus file for keyword filtering. A database dump is a file with a table configuration record and database info. This tool is an English implementation of a French paper that translates French to SQL (fr2sql) (Couderc \& Ferrero, 2015). The Graphical User Interface (GUI) interface of $\ln 2$ sql in Figure 4. Figure 4 depicts the conversion of a natural language query to a database query. 


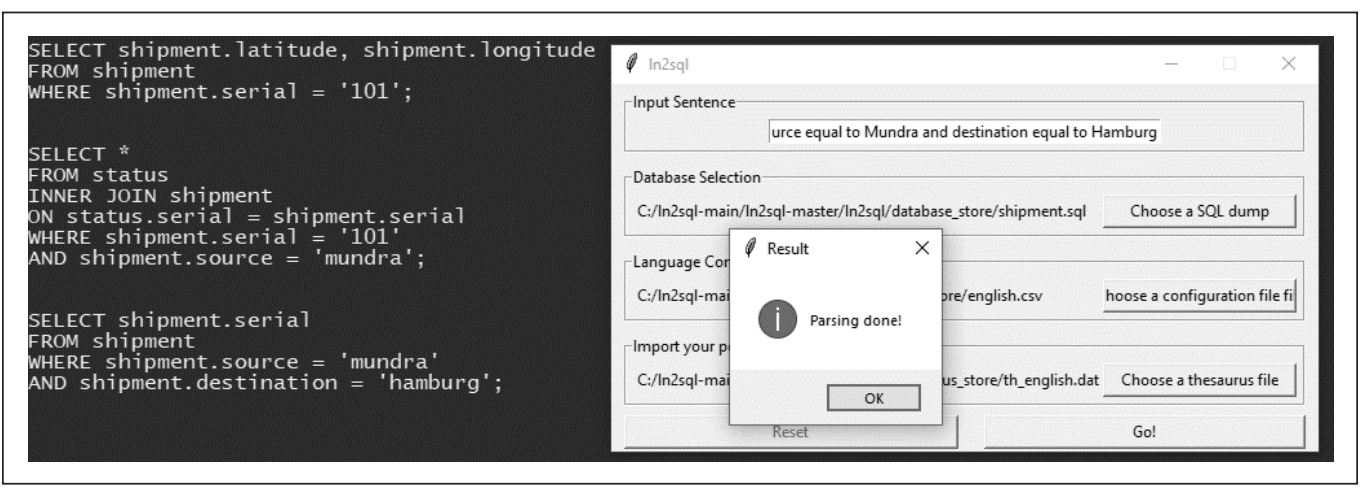

Figure 4. Converting Natural language to query language

\section{RESULTS AND DISCUSSION}

As the output from the previous step, we have a database query converted from natural language. After query formation, it is executed to generate the necessary data by the DBMS. This study offers an environment in which a user may ask a question in English, French, Turkish which will be translated into English and interpreted by multiple modules to form an equivalent SQL query that supports naïve users to find a more manageable solution and collaborative. It entirely depends on the use cases, and the applications could be limitless. The Google API can collect data, generate the vector from the database file, and display it on Google MAP view on any GUI platform. The plotting can also be done by using various other libraries on the R platform if, in any case, anyone does not want to consume Google API. The Google API is programmable so that the required performance can be shown in locations vs time or tracking waypoints for the desired logistics. Once the natural language query is translated into a database query, the user can also retrieve tracking information. This study allows users to ask various questions related to the shipment. Table 3 depicts the result generated from executing the natural language query on the proposed system. We have executed the system and gathered the answers for a random natural language query. The results are divided into three categories based on accuracy-High, Medium, Poor. The test case consists of users who know database concepts and related queries. The $20 \mathrm{such}$ users named as known users tested the system by asking two questions each using speech recognition and verified the resulting outcome of the converted query. The ten such queries for a better understanding of readers are shown in Table 3.

- High: The correct SQL statement generation from natural language is marked as 'High'

- Medium: The generated SQL statements that are partially correct are marked as 'Medium'

- Poor: The wrongly generated SQL statements or failed to generate are marked as 'Poor' 
Table 3

Result generated from executing NL query on the proposed system

\begin{tabular}{|c|c|c|c|}
\hline Natural Language Query & Generated Query & Desired Result & Decision Comment \\
\hline \multirow{5}{*}{$\begin{array}{l}\text { what is status of } \\
\text { shipment of serial } 101\end{array}$} & SELECT $*$ & SELECT * & \multirow[t]{5}{*}{ Medium } \\
\hline & FROM status & FROM status & \\
\hline & INNER JOIN shipment & INNER JOIN shipment & \\
\hline & $\begin{array}{l}\text { ON status.serial = } \\
\text { shipment.serial }\end{array}$ & $\begin{array}{l}\text { ON status.serial = } \\
\text { shipment.serial }\end{array}$ & \\
\hline & $\begin{array}{l}\text { WHERE shipment.serial } \\
\text { = OOV; }\end{array}$ & $\begin{array}{l}\text { WHERE shipment.serial } \\
=101\end{array}$ & \\
\hline \multirow{3}{*}{$\begin{array}{l}\text { what is the latitude and } \\
\text { longitude of shipment } \\
\text { having serial equal to } \\
101\end{array}$} & $\begin{array}{l}\text { SELECT shipment.latitude, } \\
\text { shipment.longitude }\end{array}$ & $\begin{array}{l}\text { SELECT shipment.latitude, } \\
\text { shipment.longitude }\end{array}$ & \multirow[t]{3}{*}{ High } \\
\hline & FROM shipment & FROM shipment & \\
\hline & $\begin{array}{l}\text { WHERE shipment.serial } \\
=\text { ' } 101 \text { '; }\end{array}$ & $\begin{array}{l}\text { WHERE shipment.serial } \\
=\text { ' } 101 \text { '; }\end{array}$ & \\
\hline \multirow{4}{*}{$\begin{array}{l}\text { tell me the serial of } \\
\text { shipment having source } \\
\text { equal to Mundra and } \\
\text { destination equal to } \\
\text { Hamburg }\end{array}$} & SELECT shipment.serial & SELECT shipment.serial & \multirow[t]{4}{*}{ High } \\
\hline & FROM shipment & FROM shipment & \\
\hline & $\begin{array}{l}\text { WHERE shipment.source } \\
=\text { 'mundra' }\end{array}$ & $\begin{array}{l}\text { WHERE shipment.source } \\
=\text { 'mundra' }\end{array}$ & \\
\hline & $\begin{array}{l}\text { AND shipment.destination } \\
=\text { 'hamburg'; }\end{array}$ & $\begin{array}{l}\text { AND shipment.destination } \\
=\text { 'hamburg'; }\end{array}$ & \\
\hline \multirow{5}{*}{$\begin{array}{l}\text { tell me shipment serial } \\
\text { where source equal to } \\
\text { Mundra and destination } \\
\text { equal to Hamburg }\end{array}$} & SELECT $*$ & SELECT shipment.serial & \multirow[t]{5}{*}{ Poor } \\
\hline & FROM shipment & FROM shipment & \\
\hline & $\begin{array}{l}\text { WHERE shipment.serial = } \\
\text { 'hamburg' }\end{array}$ & $\begin{array}{l}\text { WHERE shipment.source } \\
=\text { 'mundra' }\end{array}$ & \\
\hline & $\begin{array}{l}\text { AND shipment.source = } \\
\text { 'mundra' }\end{array}$ & $\begin{array}{l}\text { AND shipment.destination } \\
=\text { hamburg; }\end{array}$ & \\
\hline & $\begin{array}{l}\text { AND shipment.destination } \\
=\mathrm{OOV}\end{array}$ & & \\
\hline \multirow[t]{3}{*}{$\begin{array}{l}\text { what is transport of all } \\
\text { export trade shipment }\end{array}$} & $\begin{array}{l}\text { SELECT shipment. } \\
\text { transport, shipment.trade }\end{array}$ & $\begin{array}{l}\text { SELECT shipment. } \\
\text { transport }\end{array}$ & \multirow[t]{3}{*}{ Medium } \\
\hline & FROM shipment; & FROM shipment & \\
\hline & & $\begin{array}{l}\text { WHERE shipment.trade = } \\
\text { 'export'; }\end{array}$ & \\
\hline \multirow{3}{*}{$\begin{array}{l}\text { tell me shipment latitude } \\
\text { and longitude where } \\
\text { serial equal to } 101\end{array}$} & List index out of range & $\begin{array}{l}\text { SELECT shipment.latitude, } \\
\text { shipment.longitude }\end{array}$ & \multirow[t]{3}{*}{ Poor } \\
\hline & & FROM shipment & \\
\hline & & $\begin{array}{l}\text { WHERE shipment.serial } \\
=\text { ' } 101 \text { '; }\end{array}$ & \\
\hline
\end{tabular}


Table 3 (continue)

\begin{tabular}{|c|c|c|c|}
\hline Natural Language Query & Generated Query & Desired Result & Decision Comment \\
\hline \multirow{6}{*}{$\begin{array}{l}\text { tell me status of } \\
\text { shipment having serial } \\
\text { equal to } 101 \text { and source } \\
\text { equal to Mundra }\end{array}$} & SELECT * & SELECT * & \multirow[t]{6}{*}{ High } \\
\hline & FROM status & FROM status & \\
\hline & INNER JOIN shipment & INNER JOIN shipment & \\
\hline & $\begin{array}{l}\text { ON status.serial }= \\
\text { shipment.serial }\end{array}$ & $\begin{array}{l}\text { ON status.serial }= \\
\text { shipment.serial }\end{array}$ & \\
\hline & $\begin{array}{l}\text { WHERE shipment.serial } \\
=\text { ' } 101 \text { ' }\end{array}$ & $\begin{array}{l}\text { WHERE shipment.serial } \\
={ }^{\prime} 101 \text { ' }\end{array}$ & \\
\hline & $\begin{array}{l}\text { AND shipment.source = } \\
\text { 'mundra'; }\end{array}$ & $\begin{array}{l}\text { AND shipment.source }= \\
\text { 'mundra'; }\end{array}$ & \\
\hline \multirow{2}{*}{$\begin{array}{l}\text { tell me serial of all } \\
\text { shipment }\end{array}$} & SELECT shipment.serial & SELECT shipment.serial & \multirow[t]{2}{*}{ High } \\
\hline & $\begin{array}{l}\text { FROM shipment; } \\
\text { SELECT } *\end{array}$ & $\begin{array}{l}\text { FROM shipment; } \\
\text { SELECT * }\end{array}$ & \\
\hline tell me all the status & FROM status; & FROM status; & High \\
\hline \multirow{3}{*}{$\begin{array}{l}\text { What is latitude and } \\
\text { longitude of all shipment } \\
\text { having country equal to } \\
\text { India }\end{array}$} & $\begin{array}{l}\text { SELECT shipment.latitude, } \\
\text { shipment.longitude }\end{array}$ & $\begin{array}{l}\text { SELECT shipment.latitude, } \\
\text { shipment.longitude }\end{array}$ & \multirow[t]{3}{*}{ High } \\
\hline & FROM shipment & FROM shipment & \\
\hline & $\begin{array}{l}\text { WHERE shipment.country } \\
\text { = 'india'; }\end{array}$ & $\begin{array}{l}\text { WHERE shipment.country } \\
=\text { 'india'; }\end{array}$ & \\
\hline
\end{tabular}

The test set accuracy is shown in Table 4. Test set carries those questions which a known user queries. Overall, the system's accuracy is acceptable, but further investigation on query reformulation with AIS may improve accuracy. Figure 5 depicts the accuracy graph.

Table 4

Accuracy of the result generated from the proposed system

\begin{tabular}{ll}
\hline Query Category & Accuracy \\
\hline High & $60 \%$ \\
Medium & $20 \%$ \\
Poor & $20 \%$ \\
\hline
\end{tabular}

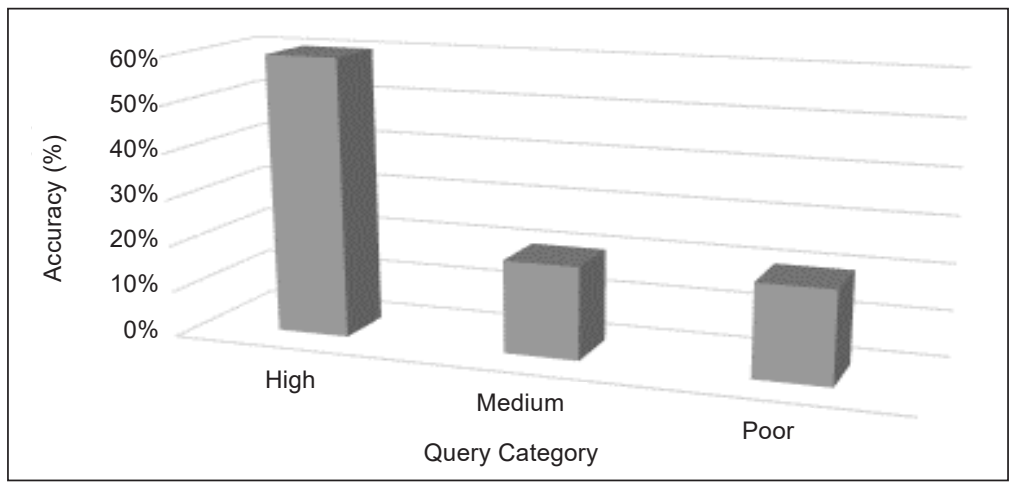

Figure 5. Accuracy of generated SQL queries with respect to desired result 


\section{THEORETICAL CONTRIBUTION}

Logistics industries are shipping millions of tons of material and goods every year. Due to different risks of damage, delay, and burglary in shipping transit, income, productivity and goods are often lost. The reliability of delivery today plays a crucial role in improving customer service. While it has been possible to track shipments in real-time via modern tracking solutions, these solutions ensure easy monitoring and analysis by providing real-time tracking of shipment location. However, the solutions remain not widely spread throughout the industry. These solutions use GPS, RFID, terrestrial systems, and logistics databases to ascertain the shipment's position at any time, but usually extremely complicated, slow, and time-consuming. This study provides an overview of the logistics tracking and management theory. The philosophy of logistics management is not a universal measure. Instead, it combines interdisciplinary knowledge and analysis that addresses the customers' motivating needs and the organisational processes to achieve broader corporate goals. There is an increasing range of methods available for tracking. However, these methods appear to complicate the problems, fail to focus on what is essential, and are burdensome for those concerned, so they do not produce lasting results. In extension to current literature, this study aims to implement AI's technological advancements using NLP to enhance the shipment tracking and management system in a logistics organisation. This research helps define the ability of NLP to track the shipment using speech recognition to provide the status of shipment in real-time and also help the stakeholders in identifying actionable insights for effective logistics network management and customer demand. This study will help the stakeholders track the shipment at ease without searching the shipment status from master data.

\section{IMPLICATION FOR PRACTICE}

The transparency of the supply chain allows a large variety of goods to be monitored during transport to obtain a simple overview of each process step. If a customer is aware of the exact location of a shipment, destination, and intended route, he can alter plans for future shipments and respond to changing circumstances immediately. Using technology to gain more knowledge of every aspect of the industry and make data-driven decisions based on information and innovation in real-time brings productivity throughout the logistics process. A combination of real-time tracking and an easy-to-use system gives naïve users the confidence they need. This study aims to enhance or bring shipment tracking to perfection in a logistics company. The study will also identify the possibilities of implementing the NLP approach and its potential for logistics tracking systems. It will give them the momentum to pursue long-term action plans and to take action to secure fast gains. The study will also assist them in the following way. 
- Transparency in delivery-allows all stakeholders to have access to information across the supply chain

- Enable end-users to make decisions in real-time

- Remove Language Barrier-caused by barriers to regional languages

- Reduced cost of trading and logistics operations and their timeframes

- Ease of doing business

\section{CONCLUSION AND FUTURE RESEARCH DIRECTIONS}

The growth of supply chain technologies has taken an exciting turn. The available technologies can change existing company operation speed, versatility, and productivity and allow innovative business models and services. In this paper, the method aims at offering a roadmap for refining transport decisions using an advanced supply chain approach. This study aims to develop an intelligent process for supervision, to track and trace the shipment. Furthermore, it provides a method that can be used to collect and communicate logistical information. This study's findings will provide valuable insight for designing more successful road-sea chain monitoring strategies focused on detected criteria and organisational challenges. Another objective of this study is to establish a partnership between the trade partners and supply chain carriers to minimise the inefficiency of physical delivery and enhance supply chain stability. However, the authors recognise that more empirical research and application are needed to validate or refute this approach's perceived advantages. The research can be further expanded through the development of an SCM-customized data analysis engine. Since the work is underway, it needs more research to ascertain its efficacy using real-time AIS data, expecting to anticipate it in the next update of this research. Our future work focuses on semantic query reformulation and query expansion and incorporates predictive analysis for routing prediction.

\section{ACKNOWLEDGEMENT}

The authors are grateful to the reviewers for providing valuable comments to improve the paper.

\section{REFERENCES}

Abbas, K., Afaq, M., Khan, T. A., \& Song, W. C. (2020). A blockchain and machine learning-based drug supply chain management and recommendation system for smart pharmaceutical industry. Electronics, 9(5), Article 852. https://doi.org/10.3390/electronics9050852

Ahkouk, K., Machkour, M., \& Antari, J. (2020). Inferring SQL queries using interactivity. In Proceedings of the 3rd International Conference on Networking, Information Systems \& Security (pp. 1-7). ACM Publishing. https://doi.org/10.1145/3386723.3387820 
Artto, K., Heinonen, R., Arenius, M., Kovanen, V., \& Nyberg, T. (1998). Global project business and the dynamics of change. Technology Development Centre Finland and Project Management Association Finland.

Bai, T., Ge, Y., Guo, S., Zhang, Z., \& Gong, L. (2021). Enhanced natural language interface for web-based information retrieval. IEEE Access, 9, 4233-4241. https://doi.org/10.1109/ACCESS.2020.3048164

Bank, W. (2019). Trade integration as a pathway to development? LAC Semiannual Report. World Bank.

Baresi, L., Meroni, G., \& Plebani, P. (2016). A GSM-based approach for monitoring cross-organization business processes using smart objects. In International Conference on Business Process Management (pp. 389400). Springer. https://doi.org/10.1007/978-3-319-42887-1_32

Betti, Q., Khoury, R., Halle, S., \& Montreuil, B. (2019). Improving hyperconnected logistics with blockchains and smart contracts. IT Professional, 21(4), 25-32. https://doi.org/10.1109/MITP.2019.2912135

Brewer, A., Sloan, N., \& Landers, T. L. (1999). Intelligent tracking in manufacturing. Journal of Intelligent Manufacturing, 10(3), 245-250. https://doi.org/10.1023/A:1008995707211

Chadil, N., Russameesawang, A., \& Keeratiwintakorn, P. (2008). Real-time tracking management system using GPS, GPRS and Google earth. In 2008 5th International Conference on Electrical Engineering/ Electronics, Computer, Telecommunications and Information Technology (Vol. 1, pp. 393-396). IEEE Publishing. https://doi.org/10.1109/ECTICON.2008.4600454

Chary, M., Parikh, S., Manini, A. F., Boyer, E. W., \& Radeos, M. (2019). A review of natural language processing in medical education. Western Journal of Emergency Medicine, 20(1), 78-86. https://doi.org/10.5811/ westjem.2018.11.39725

Chowdhary, K. R. (2020). Natural language processing. In Fundamentals of Artificial Intelligence (pp. 603649). Springer. https://doi.org/10.1007/978-81-322-3972-7_19

Christidis, K., \& Devetsikiotis, M. (2016). Blockchains and smart contracts for the internet of things. IEEE Access, 4, 2292-2303. https://doi.org/10.1109/ACCESS.2016.2566339

Christopher, M., \& Peck, H. (2004). Building the resilient supply chain. The International Journal of Logistics Management, 15(2), 1-14. https://doi.org/10.1108/09574090410700275

Clark, A., Fox, C., \& Lappin, S. (2010). The handbook of computational linguistics and natural language processing. Wiley-Blackwell. https://doi.org/10.1002/9781444324044

Couderc, B., \& Ferrero, J. (2015, June 22-25). fr2sql: Interrogation de bases de données en français [Querying databases in French]. In 22ème Traitement Automatique des Langues Naturelles (pp. 1-13). Caen, France.

Garg, R., Kiwelekar, A. W., Netak, L. D., \& Bhate, S. S. (2021a). Personalization of news for a logistics organisation by finding relevancy using NLP. In V. K. Gunjan \& J. M. Zurada (Eds.), Modern Approaches in Machine Learning and Cognitive Science: A Walkthrough: Latest Trends in AI, Volume 2 (pp. 215-226). Springer. https://doi.org/10.1007/978-3-030-68291-0_16

Garg, R., Kiwelekar, A. W., Netak, L. D., \& Bhate, S. S. (2021b). Potential use-cases of natural language processing for a logistics organization. In V. K. Gunjan \& J. M. Zurada (Eds.), Modern Approaches in Machine Learning and Cognitive Science: A Walkthrough: Latest Trends in AI, Volume 2 (pp. 157-191). Springer. https://doi.org/10.1007/978-3-030-68291-0_13 
Garg, R., Kiwelekar, A. W., Netak, L. D., \& Ghodake, A. (2021c). i-Pulse: A NLP based novel approach for employee engagement in logistics organization. International Journal of Information Management Data Insights, 1(1), Article 100011. https://doi.org/10.1016/j.jjimei.2021.100011

GEP. (2018). Artificial intelligence and its impact on procurement and supply chain: A comprehensive study. Retrieved May 9, 2020, from https://www.gep.com/white-papers/artificial-intelligence-impact-onprocurement-supply-chain

Gnimpieba, Z. D. R., Nait-Sidi-Moh, A., Durand, D., \& Fortin, J. (2015). Using internet of things technologies for a collaborative supply chain: Application to tracking of pallets and containers. Procedia Computer Science, 56, 550-557. https://doi.org/10.1016/j.procs.2015.07.251

Goll, D. C., \& Bolte, N. O. (2020). Potential analysis of track-and-trace systems in the outbound logistics of a Swedish retailer (MSc Thesis). Jonkoping University, Sweden.

He, W., Tan, E. L., Lee, E. W., \& Li, T. Y. (2009). A solution for integrated track and trace in supply chain based on RFID \& GPS. In 2009 IEEE Conference on Emerging Technologies \& Factory Automation (pp. 1-6). IEEE Publishing. https://doi.org/10.1109/ETFA.2009.5347146

Huang, W. C., Hayashi, T., Wu, Y. C., Kameoka, H., \& Toda, T. (2019). Voice transformer network: Sequenceto-Sequence voice conversion using transformer with text-to-speech pretraining. ArXiv Publishing.

Huvio, E., Grönvall, J., \& Främling, K. (2002, June 13-14). Tracking and tracing parcels using a distributed computing approach. In Proceedings of the 14th Annual Conference for Nordic Researchers in Logistics (NOFOMA'2002) (pp. 29-43). Trondheim, Norway.

Jedermann, R., Behrens, C., Westphal, D., \& Lang, W. (2006). Applying autonomous sensor systems in logistics - Combining sensor networks, RFIDs and software agents. Sensors and Actuators A: Physical, 132(1), 370-375. https://doi.org/10.1016/j.sna.2006.02.008

Kärkkäinen, M., Ala-Risku, T., \& Främling, K. (2004). Efficient tracking for short-term multi-company networks. International Journal of Physical Distribution \& Logistics Management, 34(7), 545-564. https://doi.org/10.1108/09600030410552249

Kerr, A. (1989). Information technology - Creating strategic opportunities for logistics. International Journal of Physical Distribution \& Materials Management, 19(5), 15-17. https://doi.org/10.1108/ EUM0000000000319

Kim, H. M., \& Laskowski, M. (2018). Toward an ontology-driven blockchain design for supply-chain provenance. Intelligent Systems in Accounting, Finance and Management, 25(1), 18-27. https://doi. org/10.1002/isaf.1424

Klein, T., \& Thomas, A. (2009). Opportunities to reconsider decision making processes due to auto-ID. International Journal of Production Economics, 121(1), 99-111. https://doi.org/10.1016/j.ijpe.2008.04.017

Klumpp, M., Kandel, C., \& Bioly, S. (2011). A model for mystery shipping in logistics. In 9th International Industrial Simulation Conference 2011, ISC 2011 (pp. 180-184). EUROSIS Publishing.

Ko, J. M., Kwak, C., Cho, Y., \& Kim, C. O. (2011). Adaptive product tracking in RFID-enabled largescale supply chain. Expert Systems with Applications, 38(3), 1583-1590. https://doi.org/10.1016/j. eswa.2010.07.077 
Kota, L. (2019). Artificial intelligence in logistics. Advanced Logistic Systems - Theory and Practice, 12(1), 47-60. https://doi.org/10.32971/als.2019.004

Kothris, D. (2001). Performance assessment of terrestrial and satellite based position location systems. In Second International Conference on 3 G Mobile Communication Technologies (3G 2001) (pp. 211-215). IET Digital Library. https://doi.org/10.1049/cp:20010043

Lambert, D. M., \& Cooper, M. C. (2000). Issues in supply chain management. Industrial Marketing Management, 29(1), 65-83. https://doi.org/10.1016/S0019-8501(99)00113-3

Li, J., Wang, W., Ku, W. S., Tian, Y., \& Wang, H. (2019). Spatialnli: A spatial domain natural language interface to databases using spatial comprehension. In Proceedings of the 27th ACM SIGSPATIAL International Conference on Advances in Geographic Information Systems (pp. 339-348). ACM Publishing. https:// doi.org/10.1145/3347146.3359069

Liu, D., Li, Y., \& Thomas, M. A. (2017). A Roadmap for natural language processing research in information systems. In Proceedings of the 50th Hawaii International Conference on System Sciences. HICSS Publishing. https://doi.org/10.24251/HICSS.2017.132

Loebbecke, C., \& Powell, P. (1998). Competitive advantage from IT in logistics: The integrated transport tracking system. International Journal of Information Management, 18(1), 17-27. https://doi.org/10.1016/ S0268-4012(97)00037-6

Lund, S., Manyika, J., Woetzel, J., Bughin, J., Krishnan, M., Seong, J., \& Muir, M. (2019). Globalization in transition: The future of trade and value chains. McKinsey Global Institute.

MarineCadastre.gov. (2013). AIS data handler. BOEM Publishing.

Musa, A., Gunasekaran, A., \& Yusuf, Y. (2014). Supply chain product visibility: Methods, systems and impacts. Expert Systems with Applications, 41(1), 176-194. https://doi.org/10.1016/j.eswa.2013.07.020

Neumann, M., King, D., Beltagy, I., \& Ammar, W. (2019). Scispacy: Fast and robust models for biomedical natural language processing. ArXiv Publishing.

Ruiz-Garcia, L., Steinberger, G., \& Rothmund, M. (2010). A model and prototype implementation for tracking and tracing agricultural batch products along the food chain. Food Control, 21(2), 112-121. https://doi. org/10.1016/j.foodcont.2008.12.003

Shamsuzzoha, A. H. M., Ehrs, M., Tenkorang, R. A., Nguyen, D., \& Helo, P. T. (2013). Performance evaluation of tracking and tracing for logistics operations. International Journal of Shipping and Transport Logistics, 5(1), 31-54. https://doi.org/10.1504/IJSTL.2013.050587

Sullivan, G., \& Fordyce, K. (1989). Logistics management system: Continuous flow manufacturing using knowledge based expert systems. In Proceedings of the 2nd International Conference on Industrial and Engineering Applications of Artificial Intelligence and Expert Systems-Volume 2 (pp. 520-522). ACM Publishing. https://doi.org/10.1145/67312.67313

Sultana, S., Tahsin, M., Reza, T., \& Hossam-E-Haider, M. (2016). An innovative implementation of indoor positioning system using GPS. In 2016 3rd International Conference on Electrical Engineering and Information Communication Technology (ICEEICT) (pp. 1-4). IEEE Publishing. https://doi.org/10.1109/ CEEICT.2016.7873117 
Thessen, A. E., Cui, H., \& Mozzherin, D. (2012). Applications of natural language processing in biodiversity science. Advances in Bioinformatics, 2012, 1-17. https://doi.org/10.1155/2012/391574

Töyrylä, I. (1998). Realising the potential of traceability - A case study research on usage and impacts of product traceability. In Acta Polytechnica Scandinavica Mathematics and Computing Series (Vol. 97). Helsinki University of Technology.

Wang, C., Tang, Y., Ma, X., Wu, A., Okhonko, D., \& Pino, J. (2020). fairseq S2T: Fast Speech-to-text modeling with fairseq. ArXiv Publishing.

Wang, W., Tian, Y., Wang, H., \& Ku, W. S. (2020). A natural language interface for database: Achieving transfer-learnability using adversarial method for question understanding. In 2020 IEEE 36th International Conference on Data Engineering (ICDE) (pp. 97-108). IEEE Publishing. https://doi.org/10.1109/ ICDE48307.2020.00016

Yang, G. H., Xu, K., \& Li, V. O. K. (2010). Hybrid cargo-level tracking system for logistics. In 2010 IEEE 71st Vehicular Technology Conference (pp. 1-5). IEEE Publishing. https://oi.org/10.1109/ VETECS.2010.5493655

Yuksel, M. E., \& Yuksel, A. S. (2011). RFID technology in business systems and supply chain management. Journal of Economic and Social Studies, 1(1), 53-71. https://doi.org/10.14706/JECOSS11115

Zhao, J. L., Fan, S., \& Yan, J. (2016). Overview of business innovations and research opportunities in blockchain and introduction to the special issue. Financial Innovation, 2(1), Article 28. https://doi.org/10.1186/ s40854-016-0049-2 
